\title{
La formation visant la prise en charge globale des troubles musculo-squelettiques par l'entreprise : une étude exploratoire
}

Training in order to promote global prevention and management of musculoskeletal disorders: an exploratory study

La formación dirigida a promover la prevención global y la gestión de problemas músculo-esqueléticos : un estudio exploratorio

Valérie Tremblay-Boudreault, Nicole Vézina, Denys Denis et Yannick Tousignant-Laflamme

\section{CpenEdition}

\section{Journals}

Édition électronique

URL : http://journals.openedition.org/pistes/1721

DOI : $10.4000 /$ pistes. 1721

ISSN : 1481-9384

Éditeur

Les Amis de PISTES

Édition imprimée

Date de publication : 1 mai 2011

Référence électronique

Valérie Tremblay-Boudreault, Nicole Vézina, Denys Denis et Yannick Tousignant-Laflamme, «La formation visant la prise en charge globale des troubles musculo-squelettiques par l'entreprise : une étude exploratoire », Perspectives interdisciplinaires sur le travail et la santé [En ligne], 13-1 | 2011, mis en ligne le 01 mai 2011, consulté le 19 avril 2019. URL : http://journals.openedition.org/pistes/1721 ; DOI : 10.4000/pistes. 1721

Ce document a été généré automatiquement le 19 avril 2019

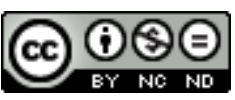

Pistes est mis à disposition selon les termes de la licence Creative Commons Attribution - Pas d'Utilisation Commerciale - Pas de Modification 4.0 International. 


\title{
La formation visant la prise en charge globale des troubles musculo-squelettiques par l'entreprise : une étude exploratoire
}

\author{
Training in order to promote global prevention and management of \\ musculoskeletal disorders: an exploratory study \\ La formación dirigida a promover la prevención global y la gestión de problemas \\ músculo-esqueléticos : un estudio exploratorio
}

Valérie Tremblay-Boudreault, Nicole Vézina, Denys Denis et Yannick Tousignant-Laflamme

\section{Introduction}

1 Les approches traditionnelles de prévention des troubles musculo-squelettiques (TMS) au travail sont souvent classées en trois niveaux d'action : 1) la prévention primaire ;2) la prévention secondaire et 3) la prévention tertiaire. La prévention primaire vise généralement l'élimination ou le contrôle des risques à la source, agissant ainsi sur les causes plutôt que sur les conséquences des TMS (ex.: modification de l'aménagement pour éliminer une posture contraignante). La prévention secondaire favorise plutôt des stratégies de dépistage pour trouver précocement les travailleurs présentant des symptômes, afin que des mesures soient prises pour favoriser le maintien en emploi et réduire l'intensité de l'exposition au risque. En ce qui concerne la prévention tertiaire, elle cible le traitement, la réadaptation, le processus de retour au travail et la prévention des rechutes des personnes présentant des TMS. Dans cet article, nous nous détachons de cette classification afin de rassembler sous le terme "prévention des TMS " toutes les actions menées par une entreprise visant à réduire l'apparition des TMS et à maintenir en emploi des travailleurs présentant des symptômes. Nous désignerons sous le terme 
«processus de retour au travail » les actions de l'entreprise pour accueillir un travailleur qui a subi une lésion.

L'objet de l'intervention étant différent entre la prévention et le processus de retour au travail, il n'est donc pas surprenant de constater que ces approches soient généralement menées en vase clos (Aptel et Vézina, 2008). Les approches de prévention sont généralement destinées à la collectivité (ex. : l'ensemble des travailleurs d'une entreprise, d'un département ou d'un poste de travail), alors que les approches favorisant le retour au travail sont davantage orientées vers les individus. De plus, les acteurs impliqués dans la prévention sont spécialisés en santé et sécurité au travail (ex. : inspecteur de la Commission de la santé et de la sécurité du travail du Québec (CSST), ergonome d'une association sectorielle paritaire) ou sont issus de l'entreprise même (comité en santé et sécurité au travail, superviseur), alors que des professionnels de la santé (ex. : médecin, ergothérapeute) sont plutôt sollicités dans le processus de retour au travail. Par conséquent, ces interventions sont généralement réalisées en parallèle, sans tirer profit des avantages de prendre en considération la prévention et le processus de retour au travail dans leur ensemble.

Pourtant, il pourrait être avantageux de créer des ponts entre ces deux approches et d'envisager une prise en charge globale des TMS. En effet, des auteurs ont rapporté que lorsque les conditions de travail sont associées aux incapacités engendrées par les TMS, un retour dans des conditions de travail inchangées (avec ou sans traitement approprié de la maladie) pourrait mener à un échec ou à des rechutes (Adler, 2006; Sanderson, 2006). Roquelaure (2007) suggère que la réduction des contraintes en milieu de travail est un pivot à la prévention précoce, et ce, tant pour éviter la survenue des TMS que pour favoriser le retour au travail. Ce constat est d'autant plus pertinent lorsqu'une prise en charge des TMS est souhaitée par l'entreprise, puisque ce sont les mêmes acteurs, plus souvent les membres d'un comité en santé et sécurité, des représentants à la prévention ou des responsables des ressources humaines, qui sont impliqués tant pour réduire les contraintes à la source que pour faciliter le retour au travail des travailleurs.

Peu d'études ont été conduites afin de favoriser la prise en charge globale des TMS par les entreprises. De plus, les outils destinés aux entreprises mettent généralement l'accent soit sur la prévention (ex. : St-Vincent et coll., 1998), soit sur le retour au travail (ex. : Stock et coll., 2005). Il ne semble donc pas exister d'outils qui visent simultanément la prévention et le retour au travail pour favoriser une prise en charge globale par l'entreprise.

5 La formation en entreprise pourrait s'avérer une porte d'entrée intéressante pour explorer la possibilité de favoriser une prise en charge globale des TMS par l'usine. En effet, Baril-Gingras, Bellemare et Brun (2007) soulignent que la formation est l'une des mesures de prévention auxquelles recourent le plus spontanément les employeurs. De plus, ces auteurs confirment la contribution positive et essentielle de la formation en santé et sécurité au travail pour la prévention au sens large. Dans une perspective de prévention des TMS, des travaux issus de l'ergonomie participative ont eu recours à la formation des acteurs en milieu de travail. À titre d'exemple, notons les interventions communément appelées les "groupes Ergo ", visant à former les acteurs de l'entreprise à l'analyse de l'activité de travail (St-Vincent et coll., 1998). Toutefois, la formation et les outils proposés dans ce genre d'intervention s'orientent vers une perspective de prévention primaire ou secondaire, sans aborder le volet tertiaire. Il devient donc 
pertinent de se demander si la formation en milieu de travail pourrait aller jusqu'à favoriser la prise en charge globale des TMS par les acteurs de l'entreprise.

Au cours d'un stage de maîtrise professionnelle en ergonomie de l'Université du Québec à Montréal, l'auteure principale a élaboré et implanté un plan de formation sur mesure, afin de favoriser la prise en charge globale des TMS par les acteurs de l'entreprise, tant du point de vue de la prévention que de celui du processus de retour au travail des personnes ayant subi une lésion musculo-squelettique. L'objectif de cet article est d'explorer et de décrire les impacts sur les différents acteurs de l'implantation d'un plan de formation qui prévoit des ponts entre ces deux approches.

\section{Méthodologie}

\subsection{Dispositif du projet}

7 Il s'agit d'une étude exploratoire descriptive basée sur l'approche d'intervention en ergonomie de Guérin et coll. (2006). L'intérêt de cette approche réside dans le fait qu'une distinction est faite entre l'analyse du travail et l'analyse de l'activité de travail. Cette dernière met en évidence les écarts entre le prescrit et le réel, c'est-à-dire dans le cas présent les écarts entre la prise en charge des TMS telle que décrite en procédure par l'entreprise et telle que réalisée sur le terrain. L'identification de ces écarts et des enjeux sous-jacents représente la base des pistes de transformation visant une prise en charge globale et plus efficace des TMS par l'entreprise. Cette approche est d'autant plus compatible avec l'objectif d'explorer et de décrire les impacts du plan de formation sur les différents acteurs que son caractère participatif favorise le recours à des entrevues et à des observations sur le terrain, permettant ainsi d'observer les impacts à la fois du point de vue des acteurs de l'entreprise et de l'intervenante en ergonomie.

\subsection{Description du contexte de l'intervention et de l'entreprise}

8 L'origine de la demande provient de la conseillère en ressources humaines de l'entreprise, qui manifestait le besoin de formation visant à accroître sa capacité de prévention et de prise en charge des TMS. L'établissement a été envoyé à la maîtrise professionnelle en ergonomie de l'Université du Québec à Montréal par un professionnel de la santé intervenant ponctuellement dans l'entreprise. Ce professionnel de la santé, un physiothérapeute, avait été engagé à titre de consultant, afin de donner aux travailleurs salariés de l'usine une formation sur la prévention des troubles musculo-squelettiques, liée plus spécifiquement à la lombalgie. Constatant que les besoins de l'entreprise dépassaient le mandat qui lui était confié, il a mis en contact la conseillère en ressources humaines de l'établissement avec le service d'ergonomie.

Il s'agit d'une filiale québécoise d'une entreprise américaine, qui fabrique et transforme le caoutchouc en des produits finis visant les marchés de la chaussure, du recouvrement de plancher résilient et du caoutchouc industriel. Les principaux procédés sont associés à une vieille machinerie exigeant un travail debout, des efforts importants de manutention et des postures contraignantes des membres supérieurs et du tronc.

10 L'entreprise survit difficilement au contexte économique précaire de ce secteur manufacturier. À cet effet, elle était en pleine implantation du lean manufacturing pour tenter d'abaisser les coûts de production et de demeurer compétitive sur le plan mondial 
au moment de l'intervention. De plus, des périodes de mise à pied régulières et le rendement aux bonis sont d'autres facteurs qui pouvaient influencer ses activités de prévention.

11 L'entreprise regroupait au moment de l'étude 294 employés, répartis dans les différents départements reliés à la production (maintenance, moulins, finition, presses et rotocures, tuiles et composites, entrepôt) et au secteur administratif. Le secteur de la production était constitué de 207 employés, œuvrant généralement sur trois quarts de travail lors de la production régulière. Les employés de production étaient représentés par l'Association des Employés du Caoutchouc (AEC) affiliée à la Centrale des syndicats démocratiques (CSD). Le secteur de la production se caractérisait principalement par une majorité de travailleurs masculins (97,6\%), un âge moyen de 46 ans [21-65 ans] et une ancienneté moyenne de 19,2 ans [1 mois-46 ans].

Comme l'entreprise faisait partie d'un secteur prioritaire, un comité en santé et sécurité (CSS) paritaire très actif était déjà en place. Il était composé des deux directeurs de production, du directeur de la maintenance, des cinq représentants à la prévention (quatre de la production et un des bureaux) et de la conseillère en ressources humaines. Il est à noter qu'en accord avec la Loi sur la santé et la sécurité du travail (LSST) en ce qui a trait à une entreprise du secteur prioritaire, un représentant à la prévention est un travailleur nommé par les membres du syndicat et a pour fonction de contribuer à la prévention dans l'entreprise, ce dernier étant libéré à cet effet un nombre d'heures donné. Il est également à noter que, dans cette usine, la formation était considérée d'emblée comme l'un des moyens de prévention les plus efficaces et la responsabilisation des employés était mise de l'avant.

\subsection{Description de l'intervention}

13 L'intervention s'est déroulée selon la démarche d'ergonomie participative proposée par Guérin et coll. (2006) et adaptée telle qu'illustrée à la figure 1.

Figure 1. Déroulement de l'intervention

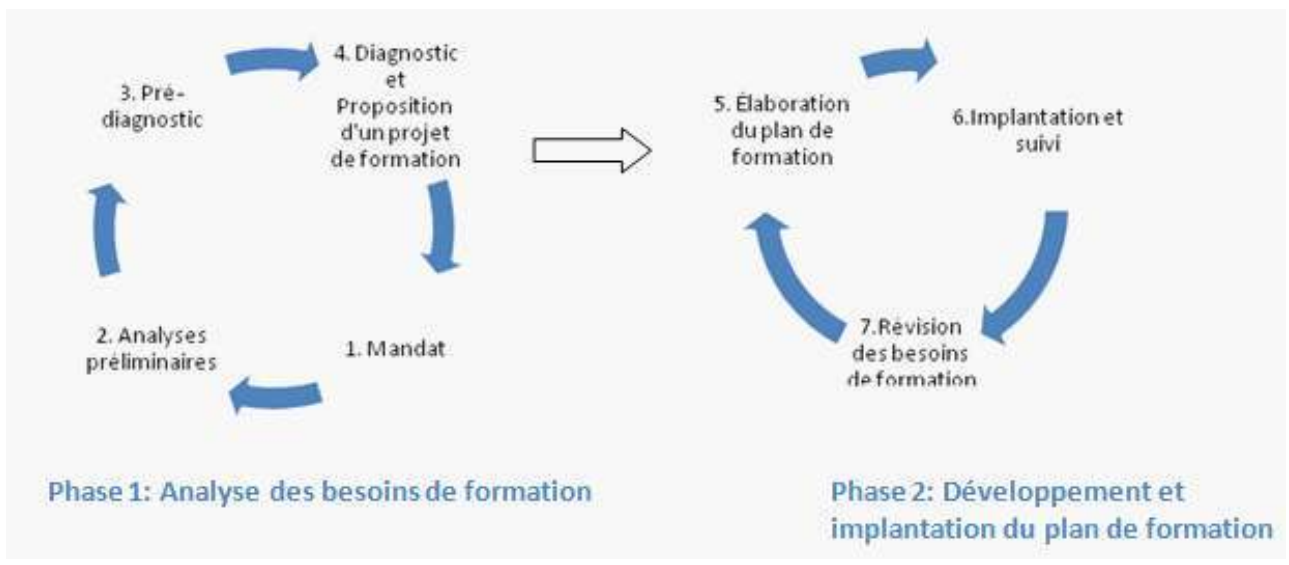

\subsubsection{Phase 1 : Analyse des besoins de formation}

L'intervenante en ergonomie a réalisé des analyses préliminaires servant à préciser le mandat et à établir le pré-diagnostic. Plusieurs sources de données ont été utilisées afin de comprendre les situations de travail en général, les principaux problèmes rencontrés, 
les procédures de prévention et de retour au travail déjà en place, ainsi que de déterminer les besoins de formation des différents acteurs de l'entreprise. Dans un premier temps, des entrevues individuelles semi-dirigées d'une durée de 30 minutes ont été tenues auprès des deux directeurs de production, du directeur de la maintenance, des représentants à la prévention $(n=4)$, de superviseurs $(n=4)$ et de la conseillère en ressources humaines. De plus, des visites des lieux de travail ont été effectuées dans chacun des secteurs de la production en compagnie de leur représentant à la prévention et de leur superviseur respectifs, totalisant trois jours d'observation. Les investigations préliminaires comprenaient également la réalisation de tournées d'inspection, appelées " audits-méthodes ", réalisées par les représentants à la prévention ou les superviseurs, ainsi que l'assistance à une réunion du comité CSS. En complément à ces investigations, divers documents de l'entreprise ont été consultés, notamment les rapports d'accident, les rapports de statistiques, le registre des accidents, les formations sur les TMS données antérieurement dans l'usine, ainsi que les manuels de procédures en santé et sécurité. De ces investigations un pré-diagnostic de la situation a pu être établi par l'intervention en ergonomie et présenté au comité en santé et sécurité de l'entreprise. Ce dernier représente la structure participative mandatée par l'employeur afin d'orienter et de faire le suivi de l'intervention. Le pré-diagnostic ayant été jugé représentatif par les membres du comité, des priorités ont été définies par le biais d'un consensus. Ces priorités représentent les pistes de transformation intégrées au plan de formation proposé par l'intervenante en ergonomie. Les principaux besoins de formation ont été établis à trois niveaux : 1) le changement des représentations reliées à la prévention et au retour au travail ; 2) la philosophie d'intégration des travailleurs à la prévention et au retour au travail et 3) le développement de compétences à l'interne. Ces éléments ont été considérés prioritaires puisqu'ils représentent des lacunes importantes à une prise en charge globale des TMS par l'entreprise.

\subsubsection{Phase 2 : Développement du matériel en cohérence avec les besoins de formation}

15 Dans un premier temps, l'intervenante a planifié le contenu du plan de formation en plusieurs blocs, qui ont été proposés à l'entreprise. Le tableau 1 présente les blocs de formation implantés, ainsi que leurs principales caractéristiques. Le contenu et les outils des différents blocs de formation étaient déterminés à partir des écrits pertinents (Conseil de la santé et de la sécurité du travail de l'Ontario (2, 3A et 3B); Stock et coll., 2005 ; St-Vincent et coll., 1998), puis adaptés en fonction des besoins établis à la phase précédente. À titre d'exemple, la procédure de maintien et de retour au travail et les outils proposés à cet effet ont été inspirés du guide de maintien et de retour au travail de Stock et coll. (2005). Des adaptations ont été apportées afin de respecter les contraintes de l'entreprise (ex.: simplification des grilles pour réduire le temps requis pour les compléter) et d'associer les différentes tâches (ex. : suivi du travailleur pendant l'arrêt de travail, définition des tâches d'assignation temporaire et suivi du travailleur pendant le retour progressif) en fonction des rôles des acteurs de l'usine. Ces nouveaux éléments venaient structurer et clarifier davantage les rôles des acteurs à ce niveau, un élément répondant au besoin de développement des compétences à l'interne. En ce qui a trait au besoin de travailler sur les diverses représentations des acteurs de l'établissement, des aspects théoriques et des exemples contextualisés à l'usine étaient intégrés dans les blocs de formation. Des points de discussion étaient prévus afin de faire ressortir les impacts 
des différentes représentations, en mettant l'accent sur les éléments qui ne permettaient pas l'application des principes de base nécessaires à la prévention des TMS, comme ce fut le cas des interventions qui ne ciblaient au départ que « la bonne méthode de travail». Ceci avait pour but de favoriser les échanges entre les participants, de confronter différents points de vue et de créer une ouverture vers un changement des perceptions, qui pouvait être poursuivi lors des suivis plus individuels. Finalement, une importance particulière était accordée à la participation active des travailleurs à tous les niveaux de prévention, tant sur le plan théorique que sur le plan pratique.

Dans un second temps, le CSS était particulièrement concerné lors de cette phase puisque, rappelons-le, il constituait la structure participative mandatée par l'employeur afin d'orienter, de valider le plan de formation et d'assurer le suivi de son implantation lors des réunions mensuelles. De plus, certains membres du CSS ont été invités à développer, en collaboration avec l'intervenante en ergonomie, la nouvelle procédure de maintien et de retour au travail afin de structurer davantage la prise en charge des TMS par l'établissement.

enu des blocs était par la suite adapté au niveau de connaissances et d'aptitudes de chacun des acteurs de l'entreprise. Pour chacun des blocs proposés en grand groupe, un exposé de type « Power Point » et des outils étaient présentés aux participants à partir de situations concrètes de l'usine préparées par l'intervenante. Les blocs visant les petits groupes étaient réalisés à partir de projets en cours dans leur département respectif et s'adressaient à des équipes de deux personnes, composées du représentant à la prévention et du superviseur pour chaque secteur de l'entreprise. Les notions sur l'analyse de l'activité de travail étaient ainsi morcelées en plusieurs petits projets déjà en cours, plutôt que de former plusieurs groupes de travail attitrés chacun à un nouveau projet de transformation de plus grande envergure. Ce format a été privilégié en raison du manque de ressources financières et des périodes régulières de mises à pied. Ces contraintes limitaient le bon déroulement des projets, notamment en rendant impossible la formation d'un groupe de travail stable dans chacun des secteurs de l'exploitation. Toutefois, un format différent a été utilisé dans le secteur des moulins, puisqu'un groupe de travail a été formé afin de participer à l'ensemble d'un projet de transformation dirigé par l'intervenante en ergonomie. Ce groupe de travail était composé à la base d'un superviseur, d'un représentant à la prévention et de trois travailleurs. Par conséquent, le bloc d'activité concrète, destiné spécifiquement à ce groupe de travail, représente l'application des notions d'analyse de l'activité de travail à travers la réalisation complète d'un nouveau projet de transformation sur un poste de travail.

Il est important de noter que ce plan de formation a été implanté de manière itérative. D'une part, les blocs de formation en grands groupes étaient présentés selon le contenu initialement prévu. Toutefois, en cours de formation, si le contenu semblait trop complexe ou à l'inverse trop de base pour les acteurs, l'intervenante tentait d'adapter le format ou le contenu aux besoins des acteurs au fur et à mesure de la formation. D'autre part, le contenu des différentes périodes de suivi en petits groupes faisait également l'objet d'adaptation, afin d'orienter les points de discussion sur la matière la plus pertinente pour le secteur de l'entreprise, ainsi qu'en fonction du niveau de compréhension des acteurs présents. Ces périodes de suivi en petits groupes s'apparentaient davantage à du " coaching " personnalisé, afin de les accompagner dans l'intégration des nouvelles notions et l'application des outils dans leur pratique au quotidien. Contrairement à une formation ponctuelle, ce type d'accompagnement auprès 
des équipes formées d'un représentant à la prévention et du superviseur d'un secteur s'inscrivait dans le temps, ce qui permettait d'approfondir le contenu des blocs précédents tout en ajoutant de nouvelles connaissances, et ce, en tenant compte du niveau de compréhension des acteurs de l'entreprise et des besoins spécifiques à chaque secteur. Ceci faisait en sorte qu'un même bloc pouvait différer d'un petit groupe à l'autre. Le contenu des blocs de formation était donc sans cesse adapté et modifié afin d'être le plus près possible des besoins des acteurs de l'entreprise et des caractéristiques de leur secteur.

Tableau 1. Caractéristiques du plan de formation

\begin{tabular}{|c|c|c|c|}
\hline Thématique du bloc et contenu abordé & $\begin{array}{l}\text { Type } \\
\text { d'approche }\end{array}$ & $\begin{array}{l}\text { Acteurs } \\
\text { concernés }\end{array}$ & Durée \\
\hline \multicolumn{4}{|l|}{ Bloc d'introduction } \\
\hline $\begin{array}{l}\text { 1. Connaissances de base sur les TMS : } \\
\text { - Ampleur des TMS dans l'entreprise } \\
\text { - Développement des TMS } \\
\text { - Principaux facteurs de risque présents } \\
\text { dans l'entreprise } \\
\text { - Aperçu de la démarche d'intervention } \\
\text { en ergonomie }\end{array}$ & $\begin{array}{l}\text { Formation } \\
\text { en grand } \\
\text { groupe } \\
(n=5-12)\end{array}$ & $\begin{array}{l}\text { Représentant à la } \\
\text { prévention } \\
\text { Superviseur } \\
\text { Conseiller RH }\end{array}$ & $\begin{array}{l}2 \text { heures } \\
\text { /département }\end{array}$ \\
\hline \multicolumn{4}{|c|}{ Blocs sur la démarche d'intervention en ergonomie } \\
\hline $\begin{array}{l}\text { 2. Introduction à la démarche / Indication } \\
\text { des priorités } \\
\text { - Introduction à la démarche } \\
\text { d'intervention en ergonomie } \\
\text { - Situation de l'audit-méthode à } \\
\text { l'intérieur de la démarche: } \\
\text { principales limites et importance de la } \\
\text { collaboration du travailleur } \\
\text { - Interprétation des statistiques du } \\
\text { département et retour sur les accidents } \\
\text { 2007 reliés aux TMS } \\
\text { - Outil pour recueillir les symptômes des } \\
\text { travailleurs } \\
\text { - Ordre de priorité des situations sur } \\
\text { lesquelles intervenir }\end{array}$ & $\begin{array}{l}\text { Formation } \\
\text { en petits } \\
\text { groupes } \\
(\mathrm{n}=2)\end{array}$ & $\begin{array}{l}\text { Représentant à la } \\
\text { prévention } \\
\text { Superviseur }\end{array}$ & $\begin{array}{l}2 \text { heures } \\
\text { /petit groupe }\end{array}$ \\
\hline $\begin{array}{l}\text { 3. Facteurs de risque et leurs déterminants } \\
\text { - Retour sur les accidents reliés aux TMS } \\
\text { et autres exemples concrets pour } \\
\text { appliquer certaines parties de la } \\
\text { démarche (risques et déterminants) } \\
\text { - L'existence du mythe de LA bonne } \\
\text { méthode de travail } \\
\text { - Exemples concrets pour pratiquer la } \\
\text { recherche des déterminants et } \\
\text { importance du rôle des travailleurs }\end{array}$ & $\begin{array}{l}\text { Formation } \\
\text { en petits } \\
\text { groupes } \\
(n=2)\end{array}$ & $\begin{array}{l}\text { Représentant à la } \\
\text { prévention } \\
\text { Superviseur }\end{array}$ & $\begin{array}{l}2 \times 2 \text { heures } \\
\text { /petit groupe }\end{array}$ \\
\hline
\end{tabular}




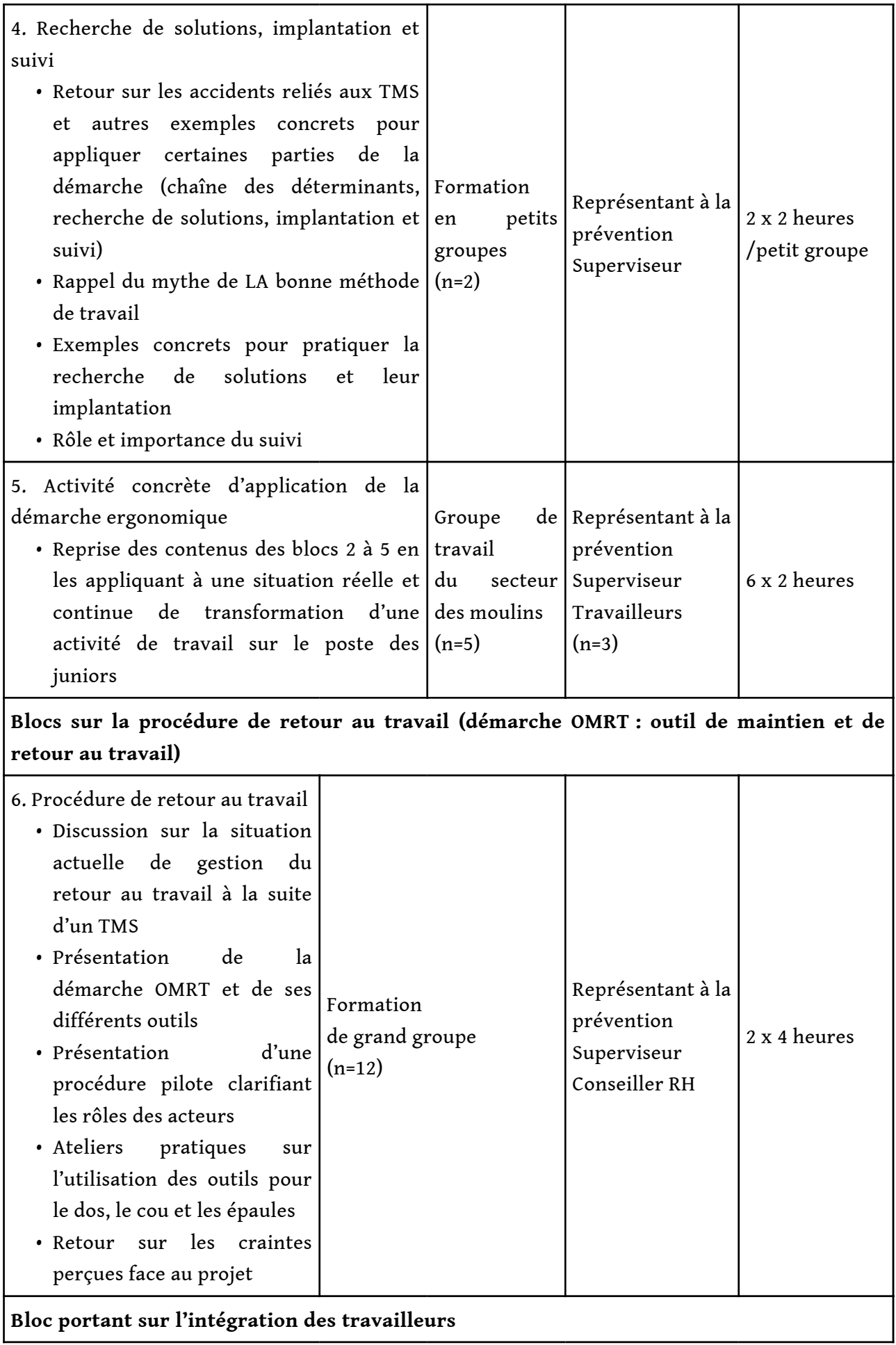




\begin{tabular}{|c|c|c|c|}
\hline $\begin{array}{l}\text { 7. Participation des travailleurs } \\
\text { à la prévention des TMS } \\
\text { Contribution souhaitée des } \\
\text { travailleurs concernant: } \\
\text { - La reconnaissance des } \\
\text { symptômes des TMS } \\
\text { - L'identification des facteurs } \\
\text { de risque et des } \\
\text { déterminants } \\
\text { - La recherche des solutions } \\
\text { et leur implantation } \\
\text { - L'adoption } \\
\text { comportement sécuritaire }\end{array}$ & $\begin{array}{l}\text { Formation de grand } \\
\text { groupe }(n=10-50)\end{array}$ & $\begin{array}{l}\text { Ensemble des } \\
\text { travailleurs à la } \\
\text { production }\end{array}$ & $\begin{array}{ll}1 & \text { heure / } \\
\text { département }\end{array}$ \\
\hline \multicolumn{4}{|l|}{ Blocs complémentaires } \\
\hline $\begin{array}{l}\text { 8. Utilité et référence à des } \\
\text { ressources externes } \\
\text { - Information du rôle des } \\
\text { différentes ressources } \\
\text { externes à consulter en } \\
\text { fonction des besoins de } \\
\text { l'entreprise }\end{array}$ & Formation individuelle & Conseiller RH & 2 heures \\
\hline $\begin{array}{l}\text { 9. Suivi personnalisé } \\
\text { d'intégration des acquis } \\
\text { - Variable selon les besoins }\end{array}$ & $\begin{array}{l}\text { Formation individuelle } \\
\text { ou en petit groupe selon } \\
\text { les besoins }\end{array}$ & $\begin{array}{l}\text { Représentant à la } \\
\text { prévention ou } \\
\text { Représentant à la } \\
\text { prévention } \\
\text { et Superviseur }\end{array}$ & $\begin{array}{l}2 \text { heures/ } \\
\text { aux deux } \\
\text { semaines } \\
\text { (les semaines } \\
\text { sans } \\
\text { formation) }\end{array}$ \\
\hline
\end{tabular}

\subsection{Collecte des données}

19 Plusieurs niveaux d'impact peuvent être attendus à la suite d'une formation (ex.: nouvelles connaissances, changement des représentations, réduction des contraintes sur certains postes de travail, implantation de nouveaux moyens de prévention, etc.). Toutefois, les variables d'intérêt présentées dans cet article porteront spécifiquement sur les besoins de formation présentés au départ. Ainsi, les impacts du plan de formation seront étudiés sous l'angle des représentations des acteurs quant à la prévention, de la philosophie d'intégration des travailleurs et du développement des compétences à l'interne.

Comme une approche itérative a été préconisée au cours de la démarche, ceci transparaît également dans le processus de collecte et d'analyse des données. En effet, la collecte des données a été réalisée au fur et à mesure des différents blocs de formation. Chaque collecte était suivie d'une analyse. Les éléments issus de ces étapes d'analyse permettaient de soulever des questions supplémentaires lors des retours effectués au moment des blocs subséquents et de corriger le tir quant au contenu et au format des formations à venir (ex. : durée, moment de la journée en fonction du quart de travail, précisions à apporter ou retour à faire sur certains aspects moins bien compris lors des blocs précédents). 
21 Ainsi, des entrevues de groupe semi-dirigées à la fin de chaque bloc de formation ont été tenues afin d'obtenir la rétroaction des participants quant aux aspects positifs, aux appréhensions et aux difficultés potentielles envisagées en référence aux procédures et outils proposés. Ces rétroactions étaient également propices pour documenter les représentations des acteurs quant à la prévention, ainsi que les éléments liés à la philosophie d'intégration des travailleurs. Une période de 15 à 20 minutes était généralement consacrée à ces échanges de groupe en fonction des questions prévues dans le canevas d'entrevue.

L'intervenante a également bénéficié de plus de 50 heures d'observations au cours des activités de formation et de suivi pendant lesquelles les acteurs étaient soumis à des situations pratiques, ainsi que lors des réunions mensuelles du CSS auxquelles l'intervenante assistait. Les changements de représentations, les facilitateurs, les difficultés rencontrées par les acteurs ou l'intervenante étaient inscrits par cette dernière dans un journal de bord prévu à cet effet.

23 Finalement, au terme de l'intervention, des entrevues individuelles semi-dirigées d'une durée de 30 minutes ont été à nouveau tenues avec les deux directeurs de production, le directeur de la maintenance, les représentants à la prévention $(n=4)$, les superviseurs $(n=4)$ et la conseillère en ressources humaines. La rétroaction a également été obtenue à deux reprises en groupe, soit lors de la dernière rencontre du CSS et lors de la rencontre avec le comité de direction.

\subsection{Analyse des données}

$24 \mathrm{Au}$ terme de chaque rencontre, individuelle ou de groupe, une fiche synthèse (Miles et Huberman, 2003) était complétée par l'intervenante en ergonomie. Le contenu faisait l'objet d'une validation auprès des participants au début du bloc de formation subséquent. Il suffisait qu'un élément soit nommé une seule fois par l'un des acteurs afin qu'il soit inscrit sur la fiche synthèse. Le contenu des fiches synthèses faisait l'objet d'une validation auprès des participants au début du bloc de formation subséquent (individuelle ou de groupe). Une fiche synthèse était également complétée avec les données du journal de bord pour chaque grand volet de formation. Les éléments similaires étaient d'abord regroupés en fonction des besoins de formation. Ainsi, pour chaque besoin de formation, des sous-thèmes ont pu être déterminés. Les informations ainsi reclassées ont été validées par le comité d'encadrement de l'étudiante à la maîtrise.

\section{Résultats}

Les impacts du plan de formation ont été regardés sous l'angle des trois grands thèmes attendus : 1) l'évolution des perceptions associées à la prévention; 2) la philosophie d'intégration des travailleurs à la prévention et 3) le développement des compétences à l'interne. Un quatrième thème a également émergé au cours de l'intervention : 4) l'appui de ressources externes pour désamorcer les craintes reliées aux conséquences possibles des actions préventives sur les travailleurs. 


\subsection{Changement des représentations associées à la prévention et au retour au travail}

l'existence d'une seule et bonne méthode de travail pour chaque tâche à accomplir. Au départ, les travailleurs s'attendaient à ce que l'ergonome détermine «LA bonne méthode de travail » à chacun des postes qui faisaient l'objet d'une discussion. Au fur et à mesure que le plan de formation s'implantait, les acteurs de l'entreprise ont commencé à considérer des facteurs de risques, des déterminants et des solutions qui allaient au-delà de la méthode de travail. À titre d'exemple, l'un des superviseurs a mentionné au cours d'une entrevue qu'il ne pouvait pas concevoir, avant les formations, agir autrement que sur la méthode de travail afin de réduire les accidents. Pour ce superviseur, «tous les accidents étaient reliés à la méthode de travail et c'est sur ça qu'on pensait devoir agir, comme on fait avec nos audits-méthodes, mais je comprends maintenant que ce n'est pas suffisant ». Cette vision initiale était partagée par plusieurs collègues dans l'usine à tous les niveaux hiérarchiques. La modification des propos tenus lors des réunions du comité en santé et sécurité et des rencontres du groupe de travail a permis de confirmer que ce changement était partagé par plus d'un acteur concerné en prévention. Si le changement de perception se répercute sur la façon d'analyser un accident pour ce superviseur, des nuances sont perceptibles entre les groupes d'acteurs. Pour les travailleurs salariés, ce changement de perception se manifestait à travers le sentiment de «se sentir responsable » de l'accident. La méthode de travail devenait donc un élément de la chaine causale plutôt que l'élément central. De façon similaire aux superviseurs, la découverte de la chaîne des déterminants a transformé la vision des représentants à la prévention des audits-méthodes, un moyen de prévention pouvant maintenant agir au-delà de la méthode de travail. Finalement, c'est à travers les préoccupations financières que ce changement de perception a pu être perçu par les membres de la direction. S'ils sont conscients de l'importance d'agir au-delà de la méthode de travail, ils sont aussi tenus de s'engager dans des projets qui assurent un retour rapide sur l'investissement. Bien que la perception de l'existence d'UNE bonne méthode de travail soit encore véhiculée dans l'entreprise, le plan de formation semble avoir réduit ce frein important à la prise en charge globale des TMS.

Un second changement a été rapporté de façon commune par les superviseurs, la conseillère en ressources humaines et un des directeurs de production. Le plan de formation leur a permis de jeter un regard nouveau sur les travailleurs en retour au travail. Pour eux, le travailleur en retour progressif ne représentait plus un " poids » pour l'entreprise, ils pouvaient maintenant envisager ce dernier comme «un membre de l'équipe de production ». Il est important ici de rappeler que la procédure habituelle de maintien ou de retour au travail en vigueur avant le plan de formation faisait en sorte que la gestion de ces cas retombait sous la responsabilité de la conseillère en ressources humaines, qui leur attribuait des tâches de bureau. Les superviseurs ne voyaient pas l'intérêt à leur trouver d'autres tâches sur le plancher, puisqu'« ils finissaient toujours par déranger leurs collègues ». Un directeur de production a d'ailleurs souligné que son changement de représentation envers les travailleurs en retour au travail constituait l'impact le plus marquant pour lui, à la suite du plan de formation. Encore une fois, il serait faux de dire que le plan de formation a entraîné la disparition de ce mode de

Perspectives interdisciplinaires sur le travail et la santé, 13-1 | 2011 
pensée, mais il a, à tout le moins, contribué à réduire un autre frein à la prise en charge globale des TMS associé aux représentations de la prévention et du retour au travail.

\subsection{Philosophie d'intégration des travailleurs} prévention, qui avait remarqué une posture contraignante pour décoller des tuiles de plancher de la pile de tuiles, avait initialement recommandé au travailleur de modifier sa posture pour être plus sécuritaire. Après avoir refait le même audit-méthode en adoptant une approche de collaboration avec le travailleur et en appliquant les étapes de la démarche ergonomique, il a été possible de remonter la chaîne des déterminants et trouver que la concentration d'un produit antiadhésif était en quantité insuffisante dans la préparation du caoutchouc. En effet, ce produit déterminait le niveau d'adhérence entre les tuiles, ce qui influençait directement la force requise et la posture des travailleurs pour décoller les tuiles des piles. Le problème a pu être repéré et corrigé à la source. Le représentant à la prévention d'un autre secteur a réalisé que sans l'appui des travailleurs, une solution pouvait difficilement être implantée. Il a fait ce constat une première fois en faisant l'expérience d'implanter un tapis de caoutchouc à un poste, ce dernier étant constamment jeté aux ordures jusqu'à ce qu'il prenne le temps de faire une tournée auprès de ses collègues, afin de discuter de l'usage du tapis de caoutchouc au poste de travail. Ce représentant à la prévention a pu constater qu'il a été encore plus facile d'implanter une surface d'appui lorsque plusieurs travailleurs avaient participé à la recherche de la solution. 
ne participation active différente des travailleurs s'est également avérée positive pour la gestion du retour au travail. La conseillère en ressources humaines, les superviseurs et les représentants à la prévention ont soulevé que la procédure de retour au travail était «moins lourde» lorsque le travailleur prenait part à la définition des tâches qu'il pourrait accomplir, notamment en complétant les grilles d'identification des risques sur le poste de travail. De plus, selon eux, les travailleurs semblaient être plus motivés et moins en attente d'une intervention de leur part. Un représentant à la prévention et un superviseur ont d'ailleurs noté que même avec un travailleur souvent en retour au travail, qui était habituellement peu collaboratif à effectuer les tâches proposées, la participation active pour définir des tâches semblait avoir réduit les comportements de dérangement des collègues et ce travailleur semblait plus motivé à accomplir les tâches pour lesquelles il avait participé à la définition.

Finalement, la conseillère en ressources humaines a également observé un avantage à impliquer activement les travailleurs présentant des symptômes douloureux dans la recherche de solutions afin de faciliter leur maintien au travail. Ces derniers lui auraient rapporté leur appréciation quant à la possibilité de bénéficier d'une attention particulière en ce qui concerne la situation de travail et de voir que « la direction était prête à faire quelque chose pour eux et à écouter leurs idées ».

Ces exemples appliqués au maintien en emploi et au retour au travail sont d'autres manifestations des impacts d'un changement de philosophie d'intégration des travailleurs. La responsabilisation de ces derniers s'est élargie au-delà de la méthode de travail et a pris un tout nouveau sens qui semble favoriser la collaboration et l'entraide. Ces exemples démontrent une autre modulation de l'approche de prévention axée sur la responsabilisation des travailleurs, qui prend maintenant un sens plus positif favorisant davantage la prise en charge globale des TMS dans l'entreprise.

\subsection{Développement des compétences à l'interne}

Des éléments sont également ressortis en association avec le développement des compétences à l'interne. Les superviseurs et les représentants à la prévention ont soulevé que le plan de formation leur avait permis d'accéder à des outils dont plusieurs étaient utiles à différents niveaux. Ainsi, les grilles d'évaluation des risques pouvaient servir à la fois pour la prévention et pour la définition des tâches à proposer lors d'un retour au travail.

Dans un même ordre d'idées, les outils et la procédure de retour au travail proposés ont permis aux acteurs de mieux définir leur rôle respectif. À titre d'exemple, un des directeurs de production a accordé beaucoup d'importance au processus de gestion du retour au travail dans la prise en charge des TMS, le considérant comme un des effets positifs les plus importants du plan de formation étant donné que des difficultés étaient particulièrement rencontrées à cet égard dans son secteur. La conseillère en ressources humaines a, elle aussi, appuyé ces propos, en mentionnant que chacun était en mesure de savoir ce qui était attendu de lui sur le plan de la prévention et sur le plan du retour au travail, ce qui permettait un "investissement complémentaire des efforts » vers un but commun. De plus, les acteurs en entreprise ont pu s'approprier en partie un nouveau langage propre à la gestion des TMS, ce qui jetait des bases communes de communication lors des échanges concernant la prévention des TMS et le retour au travail dans l'usine. Pour la conseillère en ressources humaines, ce nouveau langage était surtout utile pour 
exprimer ses besoins ou pour mieux comprendre son interlocuteur lors des discussions avec les consultants externes ou les professionnels de la santé impliqués dans le suivi des travailleurs en arrêt de travail en raison d'un TMS. Pour les superviseurs et les représentants à la prévention, ce vocabulaire a facilité les échanges avec l'intervenante en ergonomie lors de l'application concrète des outils proposés. En ce qui a trait aux directeurs de production et au directeur de la maintenance, il leur était plus facile de participer aux discussions lors des réunions du comité en santé et sécurité au travail.

Finalement, outre les enjeux techniques, des impacts des nouvelles compétences sur le plan personnel ont également été rapportés. Un des représentants à la prévention a mentionné que les nouvelles connaissances de base sur les TMS, les outils proposés et le processus de retour au travail avaient précisé ce qui était attendu de lui. De plus, ces éléments lui avaient donné plus de confiance en lui pour intervenir auprès de ses collègues plus âgés et peu enclins à parler de prévention. Un représentant à la prévention d'un autre secteur a également témoigné d'un impact similaire lorsqu'il s'agit d'approcher un travailleur afin de réaliser un audit-méthode. Ce représentant s'est dit plus confiant et mieux outillé pour aborder le travailleur selon une approche collaborative, plutôt qu'opter pour le « rôle de police " qu'il adoptait avant le plan de formation.

Ces éléments démontrent que les outils et la procédure proposés par le plan de formation ont favorisé la clarification des rôles et une gestion plus structurée des TMS, soit l'une des lacunes relevées au départ. Toutefois, il serait faux de dire que le plan de formation a entraîné uniquement des impacts positifs. En fait, une modification importante a dû être apportée afin de contrer certains effets négatifs découlant des conséquences « normalement » attendues lorsqu'il s'agit d'accroitre le niveau de responsabilité dans un champ d'expertise qui n'est pas le sien. Ce sont ces éléments qui seront présentés à la prochaine section.

\subsection{Appui de ressources externes pour désamorcer les craintes reliées aux conséquences possibles des actions préventives sur les travailleurs}

Pour la majorité des représentants à la prévention et des superviseurs, le plan de formation a également été synonyme d'une plus grande responsabilité envers la santé des travailleurs de l'entreprise, sentiment ayant évolué en fonction des formations reçues. Le niveau de responsabilité a d'abord été soulevé par rapport aux « tâches de travail » ou à la « charge de travail » par les superviseurs et par les représentants à la prévention, et ce, dès les premiers blocs théoriques présentant en grand groupe la matière dans une perspective de prévenir la survenue des TMS. Puis, certains représentants à la prévention ont fait état d'un sentiment de « doute » à l'occasion lors des exercices de proposition de solutions pour agir sur les déterminants identifiés. Ce niveau de responsabilité s'est progressivement transformé en "crainte " lorsque le contenu des blocs de formation était appliqué au retour au travail. Lors des séances des discussions en grand groupe, les représentants à la prévention et les superviseurs ont manifesté de façon commune leurs craintes à proposer des modifications à un poste de travail, suggérer des moyens de gestion de douleur ou définir des tâches de travail en vue d'un retour au travail, par peur d'aggraver la condition des travailleurs. 
Les exemples suivants permettront d'illustrer l'évolution du niveau de responsabilités. Lors d'un exercice dirigé visant à réduire le risque de TMS à la source, un représentant à la prévention avait peur de recommander une nouvelle disposition des tuiles afin de ménager l'épaule d'un travailleur, par crainte que de nouvelles douleurs apparaissent ailleurs. Un superviseur mentionnait une crainte similaire, cette fois-ci en essayant de trouver les tâches possibles avec un travailleur lors de son retour au travail. Lors de ce même exercice, le représentant à la prévention jumelé à ce superviseur a clairement manifesté son inconfort à prendre part à de telles responsabilités. Il a mentionné que « ce rôle [dépassait] largement le cadre de [ses] fonctions ». Il a même suggéré qu'il ne devrait peut-être pas participer à cette étape de la procédure de retour au travail. Cet épisode a engendré des discussions informelles entre les superviseurs et les représentants à la prévention, leurs craintes prenant une ampleur considérable. L'effet de groupe ainsi généré a nécessité un plan d'action avec les membres de la direction, afin d'éviter que le volet de maintien et de retour au travail ne soit abandonné. Lors de la formation de groupe suivante, visant l'application des connaissances pour la gestion du retour au travail, chacune des craintes a été abordée ouvertement en présence des membres de la direction. L'intention de la direction a été clairement exposée. Ainsi, dans le cas du retour au travail, l'autorisation du médecin traitant obtenue préalablement soulageait en partie ces craintes. Toutefois, lorsqu'il s'agissait de modifier un poste pour plusieurs travailleurs, aucun médecin n'y participait. C'est pourquoi un partenariat avec une entreprise externe a été développé afin d'appuyer au besoin les acteurs de l'établissement dans leur démarche de prévention. En fait, une firme de consultants, incluant un ergonome, un ergothérapeute et un kinésiologue a été recrutée afin de venir à toutes les deux semaines aider les acteurs de l'entreprise selon leurs besoins. Ainsi, des travailleurs présentant des symptômes douloureux difficiles à contrôler par les acteurs de l'établissement étaient rencontrés par les consultants selon les besoins. De plus, l'ergonome pouvait également aider un groupe de travail à l'interne lors de la réalisation d'un projet de transformation. Ce partenariat avec une entreprise externe a notamment contribué, selon les acteurs de l'entreprise, à réduire les craintes associées à la perception des acteurs d'une plus grande responsabilité envers la santé des travailleurs de l'usine. Le soutien fourni grâce au partenariat avec une ressource externe contribuait à une meilleure compréhension des situations de travail par les acteurs de l'entreprise, jouant par conséquent sur toutes les lacunes initialement présentes concernant la prévention et le retour au travail.

\section{Discussion}

Les études recensées touchant la formation des acteurs de l'entreprise portent principalement sur l'analyse ergonomique de l'activité de travail dans un but de prévention (St-Vincent et coll., 1998) ou sur le retour au travail (Stock et coll., 2005). Ainsi, le principal apport de notre étude a été de combiner la prévention et le processus de retour au travail dans un même plan de formation afin de favoriser une prise en charge globale des TMS par l'usine. Cet apport rejoint un constat récent dans la prévention des TMS. Aptel et Vézina (2008) ont analysé trois modèles de prévention des TMS afin de comprendre pourquoi l'efficacité de la prévention des TMS demeure relative malgré les multiples connaissances développées par la recherche. Ces auteurs en sont venus à la conclusion qu'il n'y a plus de raison de scinder les différents niveaux 
traditionnels de prévention. Ils illustrent bien la complémentarité des approches de prévention et de retour au travail. La prévention se réalise à partir de l'analyse des situations de travail vécues par les travailleurs, dans le but de préserver leur santé, alors que le processus de retour au travail s'amorce avec des travailleurs dont la santé est atteinte, dans le but de les maintenir en emploi. En parallèle, Roquelaure (2007) insiste sur le fait que la réduction des contraintes des situations de travail doit être le pivot de la démarche d'une prévention précoce des TMS. En ce sens, l'analyse de l'activité de travail devient un élément qui semble rejoindre tant la prévention que le retour au travail.

Les travaux issus de l'ergonomie participative, notamment ceux portant sur les groupes Ergo, ont d'ailleurs fait de l'analyse de l'activité de travail l'élément central de la formation donnée par un ergonome aux acteurs de l'entreprise (Bellemare et coll., 2002 ; Montreuil et coll., 2004 ; St-Vincent et coll., 2000). Par conséquent, un parallèle s'impose entre notre intervention et celles découlant de cette approche. Les deux types d'interventions visent la prise en charge des TMS par les acteurs de l'entreprise, à la différence que cette prise en charge intègre l'analyse de l'activité de travail jusqu'à la gestion du retour au travail dans notre cas. Le contenu et les modes de formation sont également similaires, la théorie initiale et l'application des connaissances à partir de situations réelles étant communes aux deux approches. Tel que mentionné précédemment, certains outils proposés dans le cadre de notre intervention ont été inspirés des outils du groupe Ergo (St-Vincent et coll., 1998). Toutefois, une grande proportion de nos formations a été donnée en petits groupes, c'est-à-dire aux duos "représentant à la prévention-superviseur» de chaque secteur, morcelant ainsi les étapes de l'analyse de l'activité de travail en plusieurs petits projets différents. De plus, un seul groupe de travail, composé similairement à un groupe Ergo, a réalisé une intervention complète, comparativement à plusieurs projets de transformation lors des interventions de type groupe Ergo. Ces adaptations importantes ont été apportées afin de prendre en compte les contraintes financières et les nombreuses mises à pied qui survenaient dans l'établissement. Ceci nous amène à une autre caractéristique commune, soit l'adaptation de l'intervention au contexte de l'entreprise (Montreuil et coll., 2004). Les nombreuses adaptations effectuées pour prendre en compte ce contexte ont déjà fait l'objet d'une description dans les sections précédentes. Finalement, la participation active des acteurs de l'entreprise à tous les niveaux hiérarchiques est un autre point commun qui revêt une importance capitale dans la prise en charge des TMS par l'usine. Elle favorise la complémentarité des connaissances permettant le travail en équipe sur la conception et elle représente l'occasion d'asseoir les bases d'une coopération entre les acteurs de l'entreprise, qui se poursuivra au quotidien au-delà de l'intervention (Bellemare et coll., 2002).

En fait, qu'une prise en charge des TMS par les acteurs de l'entreprise soit souhaitée ou non, la prévention en milieu de travail passe obligatoirement par l'implication des acteurs de l'entreprise (Roquelaure, 2007). En effet, bien que différents acteurs externes à l'établissement puissent également participer à la prise en charge des TMS, selon qu'il s'agisse de réduire les risques (ex. : ergonome d'une association sectorielle paritaire) ou d'accompagner un travailleur dans son processus de retour au travail (ex.: ergothérapeute), ce sont les mêmes acteurs à l'interne de l'entreprise qui seront appelés à réaliser les investigations nécessaires, exigeant d'eux de porter plusieurs chapeaux simultanément. Dans un cas comme dans l'autre, les acteurs en entreprise sont appelés à 
réduire les contraintes des situations de travail et par conséquent à analyser l'activité de travail.

De ce point de vue, la formation des acteurs en entreprise à l'analyse de l'activité de travail devient une porte d'entrée intéressante et nécessaire afin de favoriser la prise en charge globale des TMS par l'établissement. En effet, la formation est l'une des mesures de prévention les plus spontanément utilisées par les employeurs et elle apporte une contribution nécessaire à la prévention, notamment par la mise en place de conditions propices à la prévention (Baril-Gingras, Bellemare et Brun, 2007). Bellemare et coll. (2002) rapportent également que la formation à l'analyse du travail, donnée par des ergonomes, est une condition favorisant le succès de la démarche participative. Ces auteurs précisent que la formation est un moyen pour faire évoluer les représentations que les acteurs se font des problèmes à résoudre et des solutions pour y parvenir. Des constats similaires sont repris par St-Vincent et coll. (2000), dont l'intervention appuie la formule d'accompagnement par l'ergonome, visant l'acquisition de compétences en ergonomie et l'évolution des représentations des TMS et du travail qu'ont les acteurs de l'entreprise. Selon Teiger (1993), il ne peut y avoir d'augmentation de la capacité à agir en vue du changement sans qu'il n'y ait eu au préalable une transformation des représentations pour l'action. Tel qu'observé dans ce projet, le changement des représentations est l'un des principaux effets du plan de formation. À cet égard, nous émettons l'hypothèse que ces changements ne surviennent que si la formation s'inscrit dans la durée et que le formateur externe puisse bénéficier d'un certain niveau de crédibilité. De plus, les changements de représentation semblent très liés au développement et à l'application des compétences à l'interne, ainsi qu'à la création d'un climat d'échange entre les acteurs de l'entreprise. En effet, ces éléments contribuent à une meilleure compréhension de l'activité de travail. Ces constats peuvent être repris sous l'angle des trois pôles de Daniellou (1998), qui constituent les cibles sur lesquelles un ergonome peut agir afin de favoriser les transformations des situations de travail. Ainsi, les changements de représentation observés dans l'entreprise à la suite du plan de formation s'inscrivent dans le pôle du "pouvoir penser ». Le développement des compétences à l'interne, notamment par les outils proposés, les procédures de l'analyse de l'activité de travail et de la gestion du retour au travail élaborées et le langage commun développé, correspond davantage au pôle du " pouvoir agir ». Finalement, la participation active des travailleurs à tous les niveaux de prévention s'ajoute aux efforts des autres acteurs de l'entreprise pour favoriser le troisième pôle du "pouvoir débattre ", puisque plusieurs acteurs avec des visions différentes se rencontrent afin d'échanger leurs perspectives sur la situation de travail prise en considération. En ce sens, le plan de formation semble permettre la mise en place d'éléments issus de la macro-organisation qui seront utiles et nécessaires à la prévention et au processus de retour au travail.

Dans une perspective plus générale de prévention des TMS, Roquelaure (2007) soutient que la plupart des interventions en entreprise ayant démontré une efficacité sont réalisées en quatre étapes : 1) phase de mobilisation de l'entreprise et de ses acteurs ; 2) phase d'évaluation du risque; 3) phase d'évaluation des facteurs de risque et l'identification de leurs déterminants et 4) phase de transformation des situations de travail et d'évaluation. Or, la formation des acteurs de l'entreprise représente un levier important de la phase de mobilisation (Roquelaure, 2007). De plus, il est intéressant de noter que les divers blocs de formation donnés, portant sur l'analyse de l'activité de travail, rejoignent également les trois autres phases d'une intervention de prévention 
efficace, suggérant qu'une prise en charge globale des TMS par l'entreprise pourrait être réaliste après la formation. D'ailleurs, des auteurs considèrent que l'intervention ergonomique représente un acte pédagogique en soi dont bénéficient les acteurs en entreprise (Dugué, Petit et Daniellou, à paraître à l'automne 2010), ce qui rejoint le bloc d'intégration des connaissances destiné au groupe de travail ayant participé de $\mathrm{A}$ à $\mathrm{Z}$ à un projet de transformation d'un poste de travail.

La prise en charge globale des TMS par l'établissement par le biais de la formation soulève toutefois des questions importantes. La formation étant destinée aux acteurs de l'entreprise, les effets parviendront-ils à modifier les comportements de ces derniers et qu'en est-il lorsque ceux-ci quitteront l'entreprise ? Les effets de la formation auront-ils une portée suffisamment large pour atteindre l'organisation? Qu'en est-il en ce qui concerne les cas complexes dépassant la capacité des acteurs en entreprise? Des constats issus des travaux antérieurs permettent de répondre en partie à ces questions. Il est reconnu que la formation génère des effets immédiats (ex.: acquisition de nouvelles connaissances), des effets intermédiaires (ex. : implantation de solutions pour réduire les contraintes) et des effets finaux (ex. : diminution de la prévalence des TMS) (Montreuil, 1998). Si la modification des représentations est un préalable au changement dans l'action (Teiger, 1993), la portée des effets de la formation dépend notamment de la présence d'acteurs internes en mesure de prendre en charge les activités de prévention (BarilGingras et coll., 2007). Ainsi, la présence d'un CSS actif dans l'entreprise, la présence d'un représentant à la prévention dans chacun des secteurs de l'établissement représentent des leviers importants pour assurer le succès de la formation à court ou moyen terme. Toutefois, les effets de notre intervention touchent principalement les déterminants techniques et humains, une portée plus limitée ayant été observée sur le plan de l'organisation. Ce constat est similaire à St-Vincent et coll. (2000), qui ont observé un taux d'implantation des solutions supérieur pour les projets touchant l'aménagement des postes et les aspects techniques, en comparaison avec ceux reliés à l'organisation du travail.

Il est suggéré que les effets d'un plan de formation qui seraient limités aux individus seraient également éphémères étant donné que les acteurs formés ne seront pas impliqués éternellement dans les structures préventives de l'entreprise. Il importe donc que le plan de formation apporte des effets également sur le plan de l'organisation en favorisant une action soutenue de l'entreprise (Daniellou, 2004). Ces éléments de réflexion nous amènent vers la notion de prévention durable des TMS. Ainsi, pour s'engager à plus long terme dans leur prévention, une entreprise doit être consciente de l'importance des TMS et de leurs impacts, accorder les ressources nécessaires aux modifications des contraintes du travail, prévoir le transfert des connaissances de prévention lors du roulement de personnel, exiger des acteurs de l'entreprise de tous les niveaux des comptes à rendre en matière de prévention des TMS, ainsi qu'adopter une politique de prévention des TMS et cibler des indicateurs appropriés (Daniellou, 2004). Par conséquent, l'évaluation des effets à moyen et long terme d'un plan de formation visant la prévention des TMS s'avère complexe, ne serait-ce que pour isoler l'effet de l'intervention elle-même, qui s'inscrit dans le temps et dans un contexte en constante évolution. Il faut bien comprendre que l'objectif n'était pas d'évaluer les effets de la formation du point de vue des effets finaux, mais bien de s'intéresser davantage aux processus et aux effets dits intermédiaires. Le choix des indicateurs liés aux résultats présentés dans cet article visait davantage à bonifier « en continu » la formation. Il aurait 
été intéressant d'envisager d'évaluer les effets du plan de formation dans une perspective traditionnelle de coût-efficacité ou à l'aide d'approches qualitatives rigoureuses, comme la recherche-action ou l'évaluation de programme. Cependant, l'ampleur de ces démarches dépassait largement les objectifs de la maîtrise professionnelle en ergonomie.

Finalement, la notion de prévention durable ne peut être discutée sans revenir à la notion de globalité de la prise en charge des TMS. La prévention des TMS ne peut être durable si elle n'est pas globale. Nous avons parlé de globalité lorsqu'il s'agissait d'aborder la prévention des TMS en considérant simultanément la prévention et le processus de retour au travail. Roquelaure (2007) considère la globalité en matière de facteurs biomécaniques, psychologiques, sociaux et organisationnels, mettant l'accent sur la réduction des contraintes du travail et favorisant en plus une approche pluridisciplinaire lors des cas chroniques. Aptel et Vézina (2008) soutiennent que la prise en charge des victimes de TMS passe par une double approche coordonnée visant des actions thérapeutiques globales couplées à des actions ergonomiques sur le milieu de travail. Cette importance de bien articuler la prise en charge d'un travailleur, à la fois sur le plan individuel et dans le milieu de travail, comme élément clé de la réussite (Aptel et Vézina, 2008), souligne l'intérêt d'établir un partenariat entre les acteurs de l'entreprise et des ressources externes.

En effet, les auteurs s'entendent pour dire que la présence d'un ergonome ou de tout autre expert de la démarche participative demeure un facteur de réussite important, afin d'assurer la pertinence du contenu de la formation et le maintien des effets souhaités par l'entreprise (Baril-Gingras, Bellemare et Brun, 2007). En ce sens, le développement d'un partenariat avec une entreprise externe semblait donc nécessaire afin de pallier les limites inhérentes de la formation, ainsi que l'analyse de situations plus complexes (Chicoine et coll., 2006), d'autant plus que l'entreprise évolue dans un contexte économique précaire et présente une population de travailleurs vieillissante et soumise à de nombreuses contraintes. Bellemare et coll. (2002) ont permis de constater qu'un seul des sept groupes formés pouvait accomplir de façon autonome l'ensemble des étapes de la démarche du groupe Ergo, soulevant les expériences antérieures comme un des principaux facteurs explicatifs de ces résultats. Ainsi, l'accompagnement des groupes Ergo par un ergonome d'expérience permet d'accroître petit à petit leur niveau d'autonomie, réduisant graduellement l'implication de l'intervenant externe dans la démarche d'intervention. Toutefois, St-Vincent et coll. (2000) soulignent que certains éléments, dont le vieillissement et le mode de rémunération au rendement, sont trop complexes pour être pris en charge par les groupes Ergo. Un parallèle peut être effectué avec l'élargissement constant du champ d'action en santé et sécurité. Par conséquent, les préventionnistes doivent non seulement reconnaître, corriger et contrôler les risques, ils doivent désormais anticiper, communiquer, gérer et maîtriser les évolutions législatives et technologiques (Brun, 2003). Ainsi, pour soutenir les acteurs de l'entreprise dans l'élargissement de leurs fonctions, le développement d'un partenariat avec une entreprise externe est à nouveau mis en évidence.

La formation des acteurs de l'entreprise à l'analyse de l'activité de travail apparait comme un point de départ nécessaire à l'établissement d'un langage commun entre différents acteurs qui partagent un but commun : prévenir les TMS sous tous ses aspects. 


\subsection{Forces et limites}

50 Comme discuté précédemment, la principale force de notre intervention a été de combiner la prévention et le processus de retour au travail dans un plan de formation afin de favoriser une prise en charge globale des TMS par l'usine. De plus, le caractère exploratoire et très flexible de l'intervention représente, à notre avis, une force ayant permis d'être plus près des besoins de l'entreprise, ces derniers ayant évolué et s'étant précisés au cours de l'intervention. En complément à l'approche itérative de la démarche, la grande place laissée aux acteurs de l'entreprise dans les décisions relatives au contenu et au format du plan de formation a permis d'établir un plan de formation sur mesure pour l'établissement.

51 Certaines limites sont également associées à cette étude. Tout d'abord, puisqu'il s'agissait d'explorer les impacts d'un plan de formation visant une prise en charge globale des TMS par l'entreprise, et ce, auprès d'une seule entreprise, les résultats peuvent difficilement être transposables à d'autres contextes. En effet, les activités de prévention déjà en place dans ce milieu de travail jumelées au contexte particulièrement contraignant de ce dernier rendent difficile la transférabilité des résultats à d'autres contextes. Toutefois, il est important de rappeler qu'une étude exploratoire descriptive qualitative vise plutôt la crédibilité de ses résultats. En ce sens, la description en profondeur du contexte ainsi que la validation par les acteurs de l'entreprise des synthèses présentées par l'intervenante en ergonomie, au fur et à mesure du processus, sont des exemples de mesures prises afin d'assurer la crédibilité des résultats.

De plus, comme le contexte de l'intervention était celui d'un stage de maitrise professionnelle, soit une formation appliquée visant à répondre aux besoins de l'entreprise, des méthodes moins formelles de collectes et d'analyse de données ont été choisies au détriment d'un haut niveau de rigueur, tel qu'il aurait été souhaitable pour une recherche davantage à visée systématique d'évaluation d'une intervention. En fait, le caractère exploratoire et très flexible de l'intervention semble, tel que mentionné précédemment, avoir permis d'être plus près des besoins de l'entreprise qui évoluaient et se précisaient au cours de l'intervention.

53 Finalement, il aurait été intéressant de recueillir davantage les changements de représentation des travailleurs (autres que les superviseurs et les membres du CSS, dont les représentants à la prévention), qui sont des acteurs clés dans le succès de la prévention. De plus, il semble que les impacts concrets du plan de formation sur des indicateurs cibles auraient apporté un regard complémentaire aux résultats présentés, notamment sur l'efficacité et la durabilité du plan de prévention. Il est à noter qu'un plan a été proposé aux acteurs de l'entreprise afin de cibler des indicateurs, de prévoir leur évaluation et leur suivi. Toutefois, la durée du stage ne permettait pas d'évaluer les effets à plus long terme, notamment sur le mouvement de personnel et le transfert des connaissances.

\section{Conclusion}

Un plan de formation a été développé et implanté selon une approche d'ergonomie participative, afin de promouvoir la prise en charge globale des troubles musculosquelettiques par une entreprise de caoutchouc. Les principaux impacts du plan de 
formation sur les acteurs peuvent se résumer ainsi : une meilleure compréhension de l'activité de travail. Plus précisément, quatre thèmes ont été abordés afin d'expliquer cette meilleure compréhension de l'activité de travail: 1) le changement des représentations associées à la prévention;2) l'intérêt à impliquer activement les travailleurs à tous les niveaux de prévention;3) le développement des compétences à l'interne; 4) l'appui de ressources externes pour désamorcer les craintes reliées aux conséquences possibles des actions préventives sur les travailleurs. Le principal apport de notre intervention a été de combiner la prévention des TMS et le processus de retour au travail de travailleurs atteints de TMS dans un même plan de formation, afin de favoriser une prise en charge globale des TMS par l'entreprise. L'établissement d'un partenariat avec des ressources externes semble essentiel pour l'établissement afin de pallier les limites inhérentes à la formation. D'autres études sont nécessaires afin d'évaluer plus en profondeur les impacts d'un tel plan de formation, notamment en ce qui a trait à l'efficacité et à la durabilité à moyen et long terme.

Les auteurs tiennent à remercier tous les travailleurs de l'entreprise ayant pris part aux différentes étapes de l'intervention, une reconnaissance particulière s'adressant aux membres du comité de suivi et des différents groupes de travail. Nous tenons également à remercier sincèrement les collaborateurs externes suivants qui ont contribué à la démarche: Alain-Steve Comtois, Chantal Gauvin, Jaime Lara, Patrick Ménard et Carole Roy.

\section{BIBLIOGRAPHIE}

Aptel M., Vézina N. (2008). Quels modèles pour comprendre et prévenir les TMS ? Pour une approche holistique et dynamique. $2^{\mathrm{e}}$ Congrès francophone sur les troubles musculosquelettiques : de la recherche à l'action, Groupe de recherche francophone sur les troubles musculo-squelettiques. IRSST : Montréal, $27 \mathrm{p}$.

Baril-Gingras, G., Bellemare, M., Brun, J.-P. (2007). Conditions et processus menant à des changements à la suite d'interventions en santé et en sécurité du travail : l'exemple d'activités de formation. PISTES, vol. 9, $n^{\circ} 1$ www.pistes.uqam.ca/v9n1/articles/v9n1a1.htm

Bellemare, M., Marier, M., Montreuil, S., Allard, D., Prévost, J. (2002). La transformation des situations de travail par une approche participative en ergonomie : une recherche intervention pour la prévention des troubles musculo-squelettiques. Études et recherches, IRSST, R-292, 126 p. www.irsst.qc.ca/media/documents/PubIRSST/R-292.pdf

Chicoine D., Tellier C., St-Vincent, M. (2006). Le travail à tâches variées : une démarche d'analyse ergonomique pour la prévention des TMS. Études et recherches, IRSST, RG-457, 78 p. www.irsst.qc.ca/files/documents/PubIRSST/RG-457.pdf

Conseil de la santé et de la sécurité du travail de l'Ontario. (s.d.) Série sur la prévention des troubles musculo-squelettiques - Partie 2 : Manuel de ressources des Lignes directrices de la prévention des TMS pour l'Ontario. Conseil de la santé et de la sécurité du travail de l'Ontario, 72 p. www.wsib.on.ca/files/Content/Downloadable\%20FileTMS\%20Manuel/ ManuelDeResourcesTMS.pdf 
Conseil de la santé et de la sécurité du travail de l'Ontario. (s.d.) Série sur la prévention des troubles musculo-squelettiques - Partie 3A : Trousse de prévention des TMS : le point de départ. Conseil de la santé et de la sécurité du travail de l'Ontario, $50 \mathrm{p}$. www.preventionbestpractices.org/msd_tool_3af.pdf

Conseil de la santé et de la sécurité du travail de l'Ontario. (s.d.) Série sur la prévention des troubles musculo-squelettiques - Partie 3B : Trousse de prévention des TMS : au-delà de l'essentiel. Conseil de la santé et de la sécurité du travail de l'Ontario, $49 \mathrm{p}$. www.preventionbestpractices.org/msd_tool_3bf.pdf

Daniellou F. (2004). Synthèse et allocution de clôture. In Études et documents : les conditions d'une prévention durable des TMS, p. 113-123. Éditions de l'ANACT, Lyon. www.anact.fr/portal/ $\mathrm{pls/portal/docs/1/30341.PDF}$

Daniellou F. (1998). Participation, représentation, décisions dans l'intervention ergonomique. In Actes de Journées de Bordeaux sur la pratique de l'ergonomie, eds V. Pilnière, O. Lhospital, p. 3-16, Université Victor Segalen Bordeaux 2, Bordeaux.

Dugué, B., Petit, J., Daniellou, F. (2010). L'intervention ergonomique comme acte pédagogique. PISTES, vol. 12, $\mathrm{n}^{\circ} 3,22 \mathrm{p}$. www.pistes.uqam.ca/v12n3/pdf/v12n3a2.pdf

Guérin F., Laville A., Daniellou F., Duraffourg J., Kerguelen A. (2006). Comprendre le travail pour le transformer : la pratique de l'ergonomie. Éditions du réseau ANACT, Lyon.

Miles M.B., Huberman A.M. (2003). Analyse des données qualitatives. Méthodes en sciences humaines. ( $2^{\mathrm{e}}$ édition). De Boeck, Paris.

Montreuil S., Brisson C., Trudel L. (1998). Évaluation des effets d'une formation par l'amélioration des postures adaptées lors du travail avec un ordinateur. Performances humaines et techniques, $\mathrm{n}^{\circ}$ hors série, p. 27-31.

Montreuil, S., Bellemare, M., Prévost, J., Marier, M., Allard, A. (2004). L'implication des acteurs dans l'implantation de projets d'amélioration des situations de travail en ergonomie participative : des constats différenciés dans deux usines. PISTES, vol. 6, $\mathrm{n}^{\circ} 2$. www.pistes.uqam.ca/v6n2/articles/v6n2a5.htm

Roquelaure Y. (2007). Note de problématique sur les troubles musculo-squelettiques. Préparation sur les conférences des conditions de travail. Direction générale du travail. Ministère du Travail, Paris.

Stock S., Baril R., Dion-Hubert C., Lapointe C., Paquette S., Sauvage J., Simoneau S., Vaillancourt C. et coll. (2005). Troubles musculo-squelettiques : guide et outils pour le maintien et le retour au travail. Direction de la santé publique, Montréal, 52 p. www.irsst.qc.ca/media/documents/ PubIRSST/OMRT-Fr.pdf

St-Vincent M., Chicoine D., Simoneau S. (1998). Les groupes ergo : un outil pour prévenir les LATR. Études et recherches, IRSST / ASP - Secteur fabrication de produits en métal et électriques, 95 p. www.irsst.qc.ca/fr/_publicationirsst_635.html

St-Vincent, M., Toulouse, G., Bellemare, M. (2000). Démarches d'ergonomie participative pour réduire les risques de troubles musculo-squelettiques : bilan et réflexions. PISTES, vol. $2, \mathrm{n}^{\circ} 1$. www.pistes.uqam.ca/v2n1/articles/v2n1a5.htm

Teiger C. (1993). Représentation du travail. Travail de la représentation. In Représentations pour l'action, eds A. Weill-Fassina, P. Rabardel, D. Dubois, p. 311-344. Octarès édition, Toulouse. 


\section{RÉSUMÉS}

Un plan de formation a été développé et implanté selon une approche d'ergonomie participative, afin de favoriser la prévention des troubles musculo-squelettiques et la gestion du maintien et du retour au travail par une entreprise de caoutchouc. Les impacts du plan de formation ont été observés sous l'angle des changements de représentation, de la philosophie d'intégration des travailleurs et du développement des compétences à l'interne. Le principal apport de notre intervention réside dans le fait d'avoir combiné la prévention et le retour au travail au plan de formation. Compte tenu du peu de ressources spécialisées en santé et sécurité du travail et aucune en ergonomie, une collaboration entre les acteurs internes et des ressources externes apparait essentielle pour optimiser la prise en charge globale de la prévention des TMS par l'entreprise, en particulier dans un contexte alliant des ressources financières limitées, une population vieillissante de travailleurs et un niveau de contraintes élevé.

A training program was developed and implemented using a participatory ergonomics approach in order to improve prevention of MSD and related return to work management in a specific rubber plant. The results were observed under the angle of representation changes, workers' participation to prevention and return to work and internal skill development. The main contribution of our intervention was to combine altogether prevention and return to work into the training program. Collaboration between the rubber plant's actors and external resources seems to be essential in order to capitalize on proper MSD management in an industry with high level of constraints, where financial resources are limited and employees are at high risk of MSD.

Un programa de formación fue desarrollado e implantado siguiendo un enfoque de ergonomía participativa, con el fin de favorecer la prevención de trastornos músculo-esqueléticos (TMS) y la gestión del retorno al trabajo, en una fábrica de caucho. Los impactos del plan de formación fueron observados bajo el ángulo de los cambios en las representaciones, de la filosofía de reintegración de los trabajadores y del desarrollo de competencias al interior de la empresa. El principal aporte de nuestra intervención reside en el hecho de combinar la prevención y el retorno al trabajo dentro del programa de formación. Teniendo pocos recursos especializados en salud y seguridad en el trabajo y ninguno en ergonomía, una colaboración entre los actores internos y los recursos externos parecen como esenciales para optimizar la gestión global en la prevención de TMS por la empresa, en particular dentro de un contexto de recursos financieros limitados, una población de trabajadores que envejece y un alto nivel de dificultades.

\section{INDEX}

Mots-clés : ergonomie participative, prévention des TMS, formation, retour au travail, usine de caoutchouc

Keywords : participatory ergonomics, musculoskeletal disorders prevention, training, return to work, rubber plant

Palabras claves : ergonomía participativa, prevención de trastornos músculo-esqueléticos, formación, retorno al trabajo, fábrica de caucho 


\section{AUTEURS}

\section{VALÉRIE TREMBLAY-BOUDREAULT}

Valerie.Tremblay-Boudreault@usherbrooke.ca, Département de kinanthropologie, UQAM, C.P. 8888, succursale Centre-ville, Montréal (Québec), H3C 3P8

\section{NICOLE VÉZINA}

vezina.nicole@uqam.ca, Département de kinanthropologie, CINBIOSE, Institut de santé et société, C.P. 8888, succursale Centre-ville, Montréal (Québec), H3C 3P8

\section{DENYS DENIS}

denys.denis@irsst.qc.ca, Institut de recherche Robert-Sauvé en santé et en sécurité du travail, 505, boulevard De Maisonneuve Ouest, Montréal (Québec), H3A 3C2

\section{YANNICK TOUSIGNANT-LAFLAMME}

yannick.tousignant-laflamme@usherbrooke.ca, Faculté de médecine et des sciences de la santé, École de réadaptation, Université de Sherbrooke, 3001, 12e Avenue Nord, Sherbrooke (Québec), J1H 5N4 